\title{
Radon Transform Wave field separation and MATLAB implementation Weiguo ZHANG ${ }^{1, a}$, Xinjie ZHA ${ }^{1, b}$
}

\author{
${ }^{1}$ College of Computer science and technology, Xi'an University of Science and Technology, Xi'an, \\ 710054, China \\ aemail: zhangwg@xust.edu.cn, bemail: 505639788@qq.com
}

Keywords: Radon Transform; Wave Field Separation; Least-square Inversion

\begin{abstract}
In order to offer a more effective method to separate wave field, this article studies linear and high resolution nonlinear Radon transform respectively, and discusses Radon transform from continuation and discrete. The program compiled by MATLAB can achieve linear and nonlinear Radon transform. The results show that program in this article is useful and efficient, and nonlinear Radon transform suppresses the smearing effect, greatly improves the resolution.
\end{abstract}

\section{Introduction}

Radon transform was put forward by Radon initially in 1917, which provides a mathematic base for image reconstruction in physics, astronomy, medical science, optics, molecular biology[1]. Fourier projection theory testifies the equal relationship between Radon transform and Fourier transform, and Radon transform is more effective in wave filed separation[2][3]. In three-component seismic reflection data, wave field separation is an important issue which has great influence on interpretation and imaging[4]. Radon transform transforms data from an old filed into a new filed, and separates each component in the new filed, reserves the useful section[5].

This article studies linear and high resolution nonlinear Radon transform respectively, and the program compiled by MATLAB shows that nonlinear Radon transform suppresses the smearing effect, greatly improves the resolution.

\section{Linear Radon transform and nonlinear Radon transform}

Continuous Linear Radon transform

$u(h, t)$ is seismic signals, $t$ is time, $h$ is offset, $v(\tau, p)$ is the model value in $\tau-p$ filed, $\tau$ is time intercept, $\mathrm{p}$ is slope. Anti-slant stack is as follows:

$\mathrm{u}(\mathrm{h}, \mathrm{t})=\int_{-\infty}^{\infty} v(p, \tau=t-h p) d p$

Adjoint transform is as follows:

$\tilde{v}(p, \tau)=\int_{-\infty}^{\infty} u(h, t=\tau+h p) d h$

Fourier transform transforms both sides of the two equations, gets the follow equations in frequency domain as follows:

$\mathrm{U}(\mathrm{h}, \omega)=\int_{-\infty}^{\infty} V(p, \omega) e^{i \omega p h} d p$
$\tilde{V}(\mathrm{p}, \omega)=\int_{-\infty}^{\infty} U(h, \omega) e^{i \omega p h} d h$

Substituting Eq.(3) into Eq.(4), we obtain Eq.(5) as follows:

$\tilde{V}(\mathrm{p}, \omega)=\int_{-\infty}^{\infty} V\left(p^{\prime}, \omega\right)\left(\int_{-\infty}^{\infty} e^{i \omega h\left(p-p^{\prime}\right)} d h\right) d p^{\prime}$

Convolution operator is as follows:

$\gamma(\mathrm{p}, \omega)=\int_{-\infty}^{\infty} \frac{1}{|\omega|} e^{i p h} d h=\frac{2 \pi}{|\omega|} \delta(p)$

Convolution operator is an impulse function about p, we obtain Eq.(7) as follows because of the convolution property.

$\tilde{V}(\mathrm{p}, \omega)=\frac{2 \pi}{|\omega|} V(p, \omega) * \delta(p)=\frac{2 \pi}{|\omega|} V(p, \omega)$

Inverse transform formula of the raw data is as follows:

$\mathrm{V}(\mathrm{p}, \omega)=\frac{|\omega|}{2 \pi} \tilde{V}(p, \omega)=\frac{|\omega|}{2 \pi} \int_{-\infty}^{\infty} V(p, \omega) e^{i \omega p h} d h$ 
$\mathrm{h} \in[-\mathrm{H}, \mathrm{H}]$, we obtain Eq.(9) and Eq.(10) as follows:

$$
\begin{aligned}
& \gamma(\mathrm{p}, \omega)=\int_{-H}^{H} e^{i \omega p h} d h=2 H \frac{\sin (\omega H p)}{\omega H p} \\
& \tilde{V}(\mathrm{p}, \omega)=\mathrm{V}(\mathrm{p}, \omega) * \gamma(\mathrm{p}, \omega)=2 \mathrm{H} \int_{-\infty}^{\infty} V\left(p^{\prime}, \omega\right) \frac{\sin \left(\omega H\left(p-p^{\prime}\right)\right)}{\omega H\left(p-p^{\prime}\right)} d p^{\prime}
\end{aligned}
$$

This kind of definition defines inverse transform at first, then induces the transform by the least-square inversion[6].

Discrete Linear Radon transform

The definition of discrete linear Radon transform is as follows. $d(x, t)$ is the data in $t-x$ domain, $\mathrm{m}(\mathrm{p}, \tau)$ is the data in $\tau-\mathrm{p}$ domain, $\mathrm{p}$ is slope, $\tau$ is time intercept.

$\mathrm{m}(\mathrm{p}, \tau)=\sum_{x} d(h, t=\tau+p h)$

Fourier transform transforms both sides of the two equations, gets the follow equations in frequency domain as follows:

$\mathrm{M}(\mathrm{p}, \omega)=\sum_{h} D(h, \omega) e^{i w p h}$

Eq.(12) is written in matrix form as follows:

$\mathrm{M}=\mathrm{LD}$

The definition of inverse transform is as follows:

$\mathrm{D}=L^{H} M$

$\mathrm{H}$ is conjugate transpose operator, the transform is as follows by the least-square inversion.

$\mathrm{M}=\left(L L^{H}\right)^{-1} L D$

In order to stable the inversion process, we usually introduce damping factor. The value of $\mathrm{M}$ usually between 0.1 and 1 .

$\mathrm{M}=\left(L L^{H}+\mu I\right)^{-1} L D$

Nonlinear Radon transform

The algorithm of nonlinear Radon transform is similar to linear Radon transform. Linear Radon transform superposes data according to the slanted line $t=\tau+h p, \tau$ is time intercept, $\mathrm{p}$ is slope. Nonlinear Radon transform superposes data according to the parabola $t=\tau+h^{2} q$, q is curvature parameters[7]. Thus, we can deduce nonlinear Radon transform by the same method.

\section{Radon transform flow chart}

Radon transform is used wildly in wave separation, transforms data from an old filed into a new filed, and separates each component in the new filed, reserves the useful section.

\section{Algorithm achievement}

The program is compiled by MATLAB, which is divided into five menu bar: data input, format conversion, linear Radon transform, nonlinear Radon transform, as well as help.

Transforms data from an old filed into a new filed, and separates each component in the new filed, reserves the useful section. In order to testify fidelity of Radon transform, this article uses experimental data.

There are two pulses in Figure 2a. In Figure 2b, the data is transformed from t-x domain to Radon domain. We can conclude that this program is stable, on the other hand, linear Radon transform has smearing effect. 


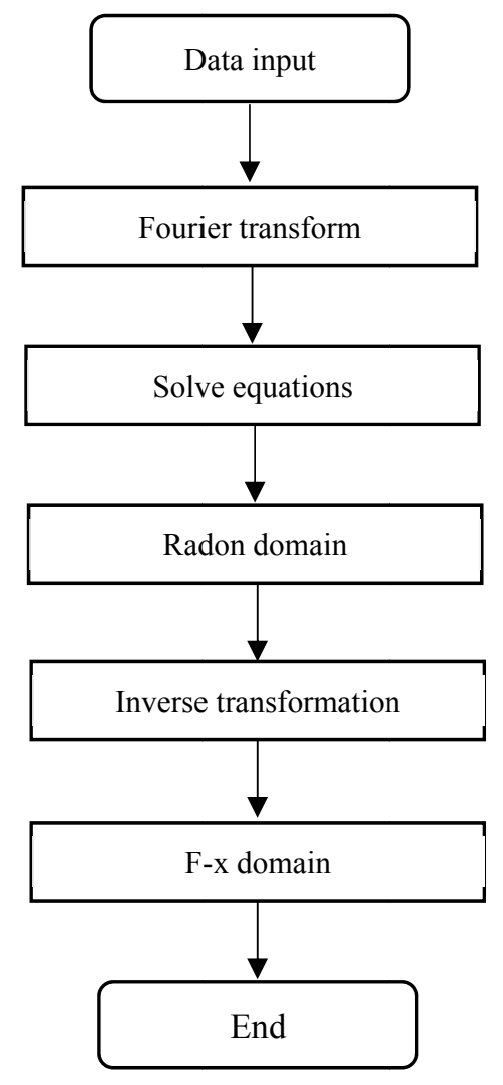

Figure 1. Radon transform flow chart

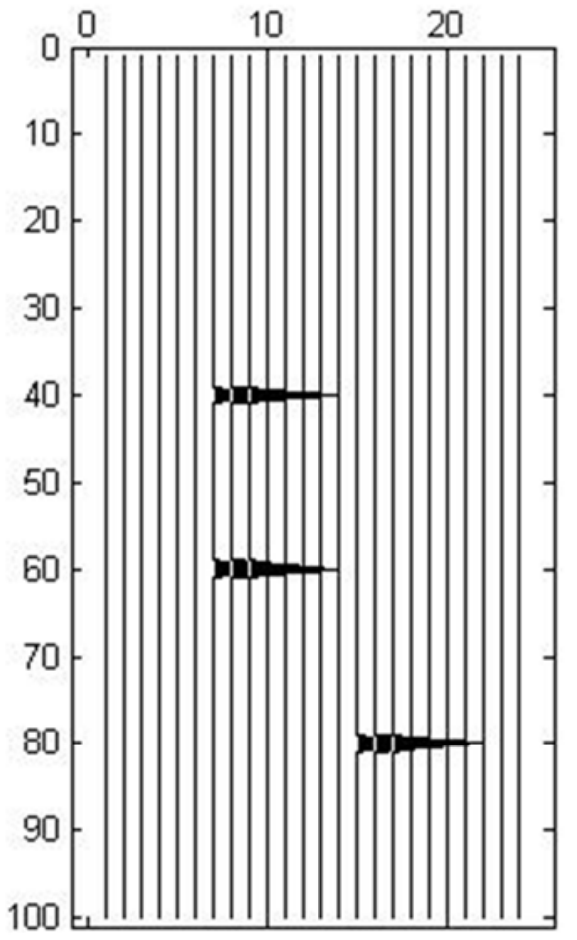

a

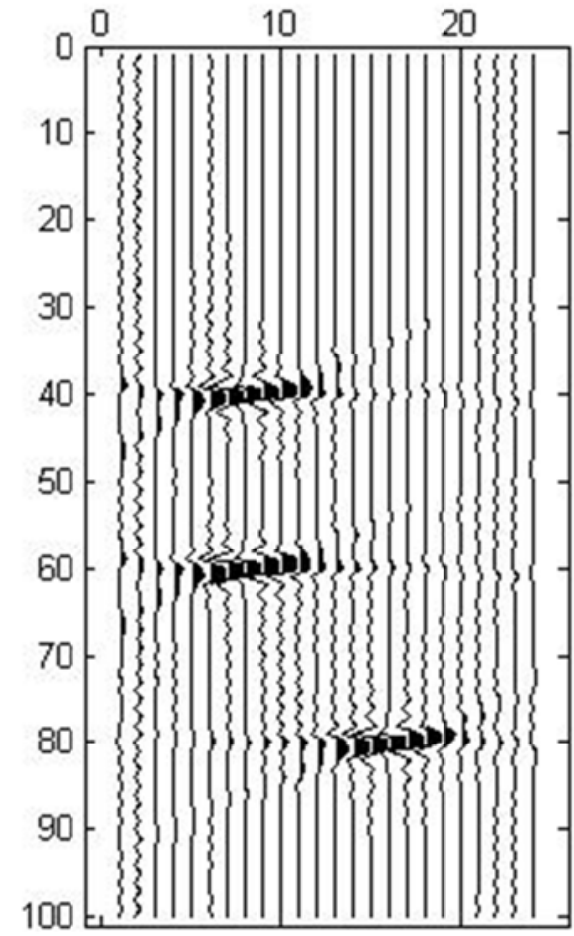

b

Figure 2. Linear Radon transform result

There are two pulses in Fig.3a. In Fig.3b, the data is transformed from t-x domain to Radon domain. Nonlinear Radon transform suppresses the smearing effect, improves the accuracy. 


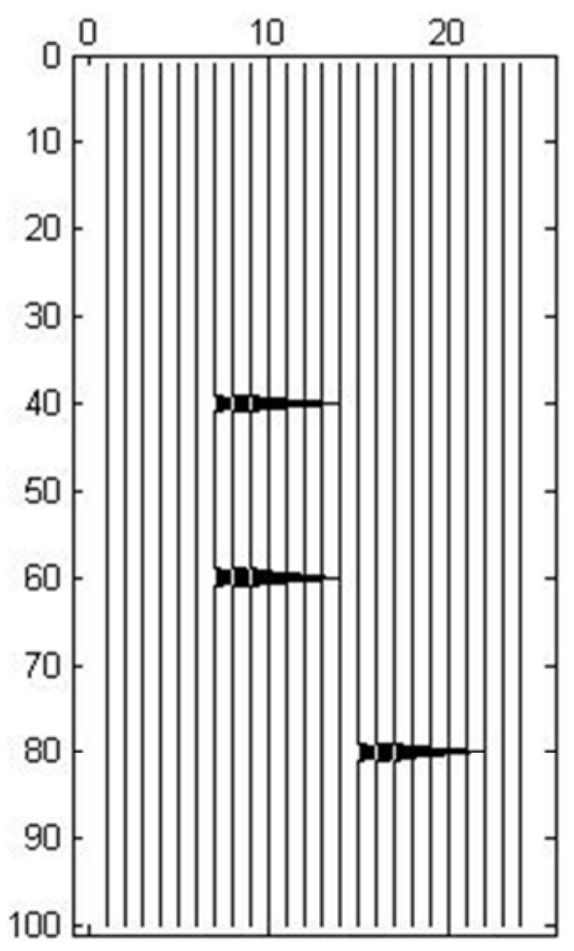

a

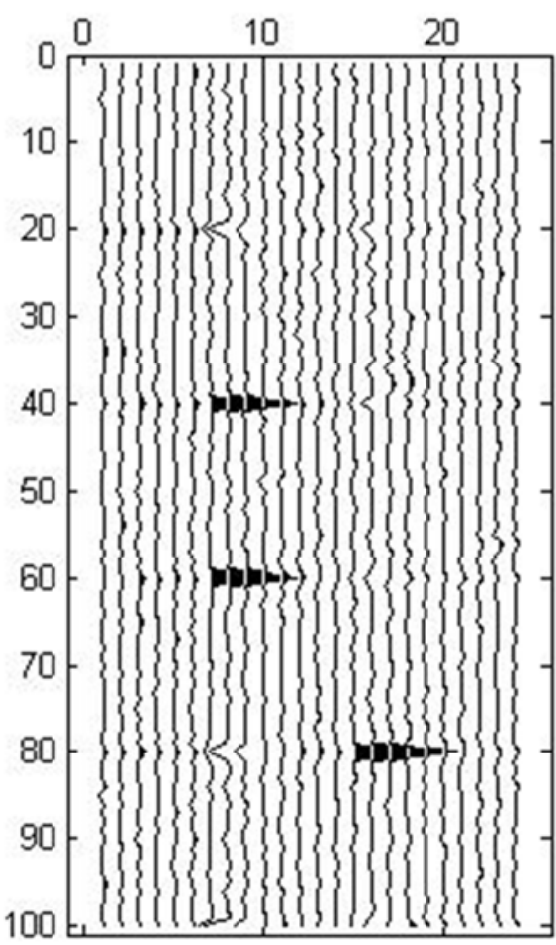

b

Figure 3. Nonlinear Radon transform result

\section{Conclusion}

This article studies linear and nonlinear Radon transform, and compiles the program based on MATLAB. We conclude that nonlinear Radon transform suppresses the smearing effect. Thus, Radon transform is a more effective method in wave separation.

\section{References}

[1] Binzhong Zhou, Stew art A. Greenhalgh. Linear and parabolic tau-p transform revisited. Geophysics, 1994, 59(7):1133.

[2] Mauricio Dino Sacchi. Aperture Compensated Radon and Fourier Transforms. The University of British Columbia, 1996:53-99.

[3] Giuseppe Marmo, Peter W. Michor, Yury A. Neretin. The Lagrangian Radon Transform and the Weil Representation. J Fourier Anal Appl, 2014: 321-361.

[4] Xiaoxue Jiang, Jun Lin, Fang Ye et al., Separation of P-P and P-SV wavefields by high resolution parabolic Radon transform, Journal of Applied Geophysics, 2015:192-201.

[5] Victor Palamodov. Reconstructive Integral Geometry. Birkhäuser Basel. 2004.

[6] Nurul Kabir M M., Verschuur D J. Restoration of missing offsets by parabolic Radon transform. Geophysical Prospecting, 1995:43-347.

[7] Kostov C. Toeplitz structure in slant-stack inversion. $60^{\text {th }}$ SEG meeting. Expanded Abstracts. 1990: 1618. 